\title{
Duration of length of stay in pneumonia: influence of clinical factors and hospital type
}

\author{
R. Menéndez*, M.J. Cremades\#, E. Martínez-Moragón ${ }^{\star}$, J.J. Soler ${ }^{+}$, S. Reyes*, M. Perpiñá*
}

Duration of length of stay in pneumonia: influence of clinical factors and hospital type. R. Menéndez, M.J. Cremades, E. Martínez-Moragón, J.J. Soler, S. Reyes, M. Perpiñá. (C) ERS Journals Ltd 2003.

ABSTRACT: Length of stay (LOS) in hospital for community-acquired pneumonia depends on the characteristics of the patient and hospital. The present study sought to identify these variables within the first $24 \mathrm{~h}$ of hospitalisation.

Patients hospitalised for pneumonia in four hospitals (one teaching and three general hospitals) had their data analysed by univariate and multivariate statististics. The variables entered were LOS, demographical characteristics, referral source, comorbidity, initial severity of illness, laboratory analyses, initial radiograph findings and antibiotic treatment regimens.

The study sample included 425 patients. The overall mortality was $8.2 \%$ and the median LOS was 9 days. Using LOS as a dependent variable, three multivariate linear regression analyses were performed with: 1) the whole cohort; 2) the low-risk classes (categories I and II of Fine); and 3) the high-risk classes (categories III, IV and V of Fine). The mathematical model identified hypoxemia, low diastolic pressure, pleural effusion, multi-lobe involvement and hypoalbuminaemia as associated with longer stays in risk classes III-V, while in the low-risk patients (I-II) only hypoxemia and pleural effusion appeared in the equation. Following adjustment for these clinical variables, the LOS remained lower in some hospitals.

Several independent clinical factors increased the pneumonia-associated length of stay with significant differences between hospitals. Hypoxemia and pleural effusions were the predictive variables of length of stay in low-risk patients and, additionally, diastolic blood pressure, multi-lobe involvement and hypoalbuminaemia were significant in the higher-risk classes III-V.

Eur Respir J 2003; 22: 643-648.
Pneumology Service of the *Hospital Universitario La Fe, ${ }^{\#}$ Hospital Francisco de Borja, "Hospital de Sagunto, and ${ }^{+}$Hospital de Requena, Valencia, Spain.

Correspondence: R. Menéndez

Servicio de Neumología

Hospital Universitario La Fe

Avda De Campanar 21

46009 Valencia

Spain

Fax: 34961973089

E-mail: rmenend@separ.es

Keywords: Community-acquired pneumonia hospitalisation

length of stay

mortality

multiple linear analysis

Received: March 82003

Accepted after revision: May 152003

This work has been supported in part by Red Respira (RTIC C03/11).
Community-acquired pneumonia (CAP) is the cause of hospitalisation for 3-5 per 1000 adults per year and with a mortality rate of $5-15 \%$. Pneumonia is the infectious disease with the highest health costs [1-4] and, since approximately one-third of all patients with CAP are treated in hospital, the resulting costs constitute a significant part of the overall direct costs of infectious diseases [5-7]. The most important component of these costs is the length of stay (LOS) in hospital and estimates indicate these costs to be higher than those of the diagnostic tests involved and the subsequent antimicrobial treatments administered [6].

There is considerable variability in LOS between hospitals. Reported findings are discordant and depend on the types of hospital in which the different studies had been conducted [8-11]. The differences might reflect variations in clinical practice preferences, hospital characteristics and patient characteristics and attitudes. Over recent years the LOS appears to have decreased from 9 to 6 days $[12,13]$ as a result of several strategies and practical guidelines that have been proposed in order to safely reduce the number of hospitalisation days [14].

The LOS is influenced by several clinical factors, such as the Pneumonia Severity Index (PSI) [15] associated comorbidity, and the presence of clinical complications. These factors have been evaluated in recent reports [9, 11, 16, 17], but todate, there has been no clear identification of the variables that determine LOS using the methods of multivariate analyses with adjustment for confounding variables. Neither have there been any studies on the possible differences in LOS between patients admitted to hospital with different grades of seriousness of the illness. Published studies have indicated that a substantial percentage of hospitalisations are comprised of patients of risk classes I-II that, despite the low probability of death ensuing if treated on an outpatient basis, nevertheless could benefit more from hospitalised treatment.

The hypothesis of the current study was that LOS is not influenced by identical variables affecting patients with different grades of initial severity of illness and that the type of admitting hospital has an influence. Hence, the objective of the present study was to identify, during the first $24 \mathrm{~h}$ following admission, the clinical factors associated with the duration of hospitalisation for CAP in patients with different grades of illness on admission (risk classes I and II versus risk classes III-IV and V). Identification of independent predictive factors of LOS would help clinicians to evaluate the need for, and the duration of, hospitalisation for community acquired pneumonia and to rationalise the patient's discharge from hospital.

\section{Patients and methods}

\section{Study subjects}

A prospective follow-up study was performed in four public hospitals, of which three are general urban hospitals of 
different sizes and one a referral (teaching) hospital, in Valencia, Spain. These were: 1) Hospital Universitari La Fe, a 900-bed teaching hospital serving a population of 400,000 and with 13 physicians specialising in lung diseases; 2) Hospital de Sagunto, a 270 bed community hospital serving a population 128,000 and with three lung specialists; 3) Hospital de Gandía, a 240-bed community hospital serving a population of 125,000 and with three lung specialists; and 4) Hospital de Requena, a 106-bed hospital serving a population of 58,000 and with two lung specialists. Patients consecutively admitted to the four hospitals during a 12-month period were included. Inclusion criteria were the presence of a chest radiograph with evidence of infiltrate and symptoms compatible with CAP. Alternative diagnoses were excluded during the follow-up period. Patients with immunosuppression, including human immunodeficiency virus infection, patients who had been hospitalised in the previous 15 days and those with tuberculosis were also excluded, as were those patients who received attention in the intensive care unit.

\section{Data collection}

A protocol was devised that sought data on demographical characteristics, comorbidity, initial evaluation of the risk class according to Fine, or the PSI [15], LOS and outcome. Data were collected as follows. In the first $24 \mathrm{~h}$, the 20 variables that comprise the prognostic scale of Fine [15] were evaluated and the patient was classified in one of the five risk classes (range, I-V). Demographical characteristics including age, gender, smoking habit and alcohol intake were recorded. Specific comorbidity details included chronic obstructive pulmonary disease (COPD), asthma, cardiac diseases, renal or hepatic diseases, diabetes mellitus, prior hospitalisation or prior CAP. Concomitant medications, such as oral or inhaled corticosteroids and previous antimicrobial treatments, were also recorded.

Initial clinical symptoms and physical signs noted were pleural pain, cough, expectoration, abrupt onset dyspnoea, and the time-lapse (in days) from symptom onset. The presence of cyanosis, blood pressure measurement, respiratory rate, level of consciousness and auscultation data were also noted. Laboratory analyses recorded leukocyte, haematocrit, plasma urea (BUN), albumin, sodium, potassium and platelet levels and blood gas measurements (arterial oxygen tension $\left(\mathrm{Pa}, \mathrm{O}_{2}\right)$, arterial carbon dioxide tension, and $\left.\mathrm{pH}\right)$ on admission. Radiograph data on admission assessed the number of lobes affected and the presence/absence of pleural effusion.

Initial antimicrobial regimens prescribed were as follows: third generation cephalosporins, third generation cephalosporins and macrolides, macrolides alone, quinolones, amoxycillinclavulanate alone or with macrolides, and other regimens. Antimicrobial treatment was classified as adhering, or not, to Spanish guidelines [18]. Adherence-to-guidelines treatment was defined as initial antimicrobial regimen consisting of third generation cephalosporins alone or with macrolides, amoxycillin-clavulanate alone or with macrolides and quinolones (third or fourth generation) alone. Other treatment regimens were defined as not adhering to the guidelines. LOS was defined as the number of days between admission and discharge.

\section{Statistical analysis}

Univariate analyses were performed using all the variables recorded on admission, the demographical characteristics, initial risk class, laboratory and radiograph data, and the number of days of hospitalisation. Pearson correlation analysis was used for data that followed a normal distribution and Spearman correlation for those that followed a nonnormal distribution. All p-values of $<0.05$ were considered statistically significant.

The variables that were found to be significant $(p<0.05)$ in the univariate analysis were introduced as independent variables in a multivariate stepwise linear regression analysis with the LOS (in days) as the dependent variable.

\section{Results}

\section{Study population}

A total of 425 patients admitted with CAP were included in the study. The demographical characteristics, comorbidity, PSI index and mortality in the four hospitals are summarised in table 1 and table 2. No differences were found between the four hospitals with respect to age, gender and previous comorbidities of the most frequent coexisting conditions, such as congestive heart failure, COPD, diabetes, renal disease and cerebrovascular disease. Active smokers were more frequent in Hospitals $\mathrm{C}$ and $\mathrm{D}(\mathrm{p}<0.02)$. Mortality expressed as a function of each initial risk class showed statistically nonsignificant differences in the four hospitals (table 2).

The percentage distributions of patients in the teaching hospital with respect to PSI were 9.6, 10.9, 24, 39.3 and 16.2 in the risk classes I, II, III, IV and V, respectively. The differences in the distributions were not statistically different $(p=0.18)$ from the corresponding percentage distributions in

Table 1.-Demographical characteristics, comorbidity and initial risk class of the patient cohort

\begin{tabular}{|c|c|c|c|c|}
\hline Characteristic & Hospital A & Hospital B & Hospital C & Hospital D \\
\hline Patients $\mathrm{n}$ & 229 & 73 & 58 & 65 \\
\hline Age yrs ${ }^{\#}$ & $69 \pm 16$ & $70 \pm 16$ & $68 \pm 17$ & $72 \pm 16$ \\
\hline Sex M/F & $65 / 35$ & $63 / 37$ & $59 / 41$ & $71 / 29$ \\
\hline Residence for the elderly $\%$ & 3.5 & 2.7 & 8.6 & 4.6 \\
\hline Alcohol \% & 16 & 7 & 19 & 21 \\
\hline Smokers $\% *$ & 14.8 & 19.2 & 24 & 25 \\
\hline COPD $\%$ & 32 & 41 & 31 & 34 \\
\hline Cardiac disease $\%$ & 33 & 18 & 26 & 23 \\
\hline Diabetes $\%$ & 22 & 18 & 22 & 14 \\
\hline Liver disease $\%$ & 5 & 4 & 7 & 8 \\
\hline CNS disease \% & 15 & 11 & 15 & 19 \\
\hline Renal disease \% & 5 & 3 & 9 & 6 \\
\hline
\end{tabular}

M: male; F: female; COPD: chronic obstructive pulmonary disease; CNS: central nervous system. ${ }^{\#}$ : data are presented as mean \pm SD. ${ }^{*}: \mathrm{p}<0.05$. 
Table 2. - Initial risk class and mortality

\begin{tabular}{|c|c|c|c|c|c|c|c|c|}
\hline \multirow[t]{2}{*}{ Risk class } & \multicolumn{2}{|c|}{ Hospital A } & \multicolumn{2}{|c|}{ Hospital B } & \multicolumn{2}{|c|}{ Hospital C } & \multicolumn{2}{|c|}{ Hospital D } \\
\hline & $\%$ total & $\%$ dead & $\%$ total & $\%$ dead & $\%$ total & $\%$ dead & $\%$ total & $\%$ dead \\
\hline PSI I & 9.6 & 0 & 5.5 & 0 & 8.6 & 0 & 1.5 & 0 \\
\hline PSI II & 10.9 & 0 & 12.3 & 0 & 13.8 & 0 & 15.4 & 0 \\
\hline PSI III & 24 & 5.5 & 23.3 & 0 & 27.6 & 0 & 7.7 & 0 \\
\hline PSI IV & 39.3 & 8.8 & 45.2 & 9.1 & 31 & 5.6 & 43.1 & 7.1 \\
\hline PSI V V & 16.2 & 21.6 & 13.7 & 30 & 19 & 27.3 & 32.3 & 14.3 \\
\hline Total & & 8.7 & & 8.2 & & 6.9 & & 7.7 \\
\hline
\end{tabular}

PSI: pneumonia severity index. ${ }^{*}$ : $\mathrm{p}=$ not significant.

the general hospitals $(5.1,13.8,19.4,40.3$ and 21.4 in the risk classes I, II, III, IV and V, respectively).

Antibiotic therapy included the administration of third generation cephalosporins in 32 patients, amoxycillin-clavulanate in 39 , quinolones in eight, macrolides in 32 and a combination of $\beta$-lactams and macrolides in 238. Other antimicrobial agents had been employed in 76 patients. Adherence to the Spanish guidelines, Sociedad Espanola de Neumología y Cirugía Torácica (SEPAR), with respect to empirical treatment was $75 \%$. Significant differences were observed in the antimicrobial regimens prescribed in the four hospitals: in Hospital B, the adherence to the SEPAR guidelines was significantly lower.

\section{Natural history of the illness and its outcome}

There was an overall mortality rate of $8.2 \%$ (35 patients). There were no significant differences in the distribution of patients according to risk class for mortality and hospital (table 2). Logistic regression analysis was performed to predict death (dependent variable) using the initial risk class (according to Fine; classes I-V) and the type of hospital as independent variables. The model selected only initial risk class as a significant predictor of mortality (odds ratio 2.6).

\section{Length of stay in hospital}

Univarite analysis. The statistical analysis of duration of hospitalisation was performed and excluded those patients who died during hospitalisation. The median LOS in the group of patients who had died was 7 days (range, 1-27 days). The LOS segregated with respect to risk class is presented in table 3. LOS was shorter in Hospital $\mathrm{D}, \mathrm{p}<0.05$. Due to the skewed distribution of LOS, nonparametric tests were used.

The LOS in surviving patients, following the initial antibiotic treatment, was lower in those treated with quinolones and amoxycillin-clavulate+macrolides $(\mathrm{p}=0.035)$ (table 4). When the data were analysed with respect to adherence to

Table 3. - Initial risk class and duration of hospitalisation at each hospital

\begin{tabular}{lrrrr}
\hline Risk class & Hospital A & Hospital B & Hospital C & Hospital D \\
\hline PSI I & $6(7 \pm 4)$ & $8(8 \pm 3)$ & $10(9 \pm 2)$ & $3(3 \pm 0)$ \\
PSI II & $8(8 \pm 3)$ & $8(8 \pm 2)$ & $9(9 \pm 4)$ & $4(5 \pm 2)$ \\
PSI III & $9(11 \pm 6)$ & $8(9 \pm 5)$ & $7(8 \pm 2)$ & $14(14 \pm 7)$ \\
PSI IV & $9(10 \pm 6)$ & $10(11 \pm 5)$ & $7(9 \pm 4)$ & $6(7 \pm 5)$ \\
PSI V & $9(11 \pm 7)$ & $8(9 \pm 4)$ & $11(10 \pm 3)$ & $7(7 \pm 3)$ \\
Total & $8(10 \pm 6)$ & $8(10 \pm 4)$ & $8(9 \pm 3)$ & $6(7 \pm 5)$ \\
\hline
\end{tabular}

Data are presented as median (mean $\pm \mathrm{SD}$ ). $\mathrm{p}<0.05$.
SEPAR guidelines, there were no significant differences.

Spearman correlation analysis was performed using all the initial variables. Age, gender, alcohol intake, smoking habit and comorbidity were not significantly correlated with LOS. Significant positive correlations were observed with the PSI index, presence of pleural effusion, number of lobes affected and BUN concentration. Significant inverse correlations were observed with the $\mathrm{Pa}, \mathrm{O}_{2}$ level on admission, concentration of albumin, presence of neoplasia and diastolic blood pressure.

Multivariate analysis. Three multivariate linear regression analyses were performed to predict the duration of hospitalisation (dependent variable). The first run included the total cohort, the second included only those patients in risk classes III, IV and V and the third included those patients in risk classes I and II. The independent variables were those found to be significant in the univariate analyses. Additional variables were initial antibiotic treatment, adherence to SEPAR guidelines, and the hospital to which the patients had been admitted (A, B, C and D: introduced into the analyses as dummy variables). The quantitative variables included were albumin concentration, diastolic blood pressure and arterial $\mathrm{Pa}, \mathrm{O}_{2}$.

The mathematical model identified six independent variables for the whole cohort and for risk classes III-V. These were albumin, pleural effusion, $\mathrm{Pa}, \mathrm{O}_{2}$ on admission, type of hospital (shorter stays in Hospitals C and D), number of lobes affected in the initial radiograph and diastolic blood pressure. The coefficients of the models and the estimation errors are presented in table 5 . The mathematical model, when only the risk classes I-II were analysed, identified hypoxemia, pleural effusion and admission to Hospital $\mathrm{D}$ as being the only variables significantly related to LOS.

\section{Discussion}

In the present study, different clinical variables were identified as being associated with LOS when the patient population was analysed on the basis of the initial risk class.

Table 4.-Duration of hospitalisation in relation to initial antibiotic treatment

Antibiotic regimen

Days of stay

Third generation Cephalosporin

Amoxycillin-clavulanic acid

Amoxycillin-clavulanic acid and macrolide

Macrolide

Quinolone

Others

$8(10 \pm 5)$

$9(10 \pm 7)$

$6(7 \pm 3)$

$8(8 \pm 3)$

$6(7 \pm 4)$

$8(9 \pm 4)$

Data are presented as median (mean $\pm \mathrm{SD})$. $\mathrm{p}=0.03$. 
Table 5.-Predictive factors of length of duration of hospitalisation

Variable in the model

Coefficient

\begin{tabular}{lccc}
\cline { 2 - 4 } & $\begin{array}{c}\text { Fine } \\
\mathrm{I}-\mathrm{II}^{\#}\end{array}$ & $\begin{array}{c}\text { Fine III, IV } \\
\text { and } \mathrm{V}^{\boldsymbol{*}}\end{array}$ & $\begin{array}{c}\text { Fine } \\
\mathrm{I}^{+}-\mathrm{V}^{+}\end{array}$ \\
\hline Constant & 8.8 & 18.3 & 18.4 \\
Albumin $\mathrm{mg} \cdot \mathrm{dL}^{-1}$ & & -1.5 & -1.4 \\
Pleural effusion & 2.5 & 3.2 & 3.2 \\
$\mathrm{~Pa}, \mathrm{O}_{2} \mathrm{~mm} \cdot \mathrm{Hg}^{\S}$ & -0.058 & -0.075 & -0.08 \\
$\mathrm{Hospital} \mathrm{D}$ & -3.9 & -3.07 & -3.2 \\
Number of lobes involved & & 1.59 & 1.4 \\
Diastolic blood pressure & & -0.059 & -0.06 \\
Hospital C & & -0.07 & -2.3 \\
\hline
\end{tabular}

$P \mathrm{a}, \mathrm{O}_{2}$ : arterial oxygen tension. ${ }^{*}$ : correlation coefficient $(\mathrm{r})=0.55 ; \mathrm{SE}=2.5$, f value $=8.7 ;{ }^{\natural}: \mathrm{r}=0.442, \mathrm{SE}=4.9, \mathrm{f}$ value $=8.3 ;{ }^{+}: \mathrm{r}=0.471, \mathrm{SE}=4.6$; $\mathrm{f}$ value $=12.4$; §: $\mathrm{mm} \cdot \mathrm{Hg} \times 0.133=\mathrm{kPa}$.

In the low-risk sub-groups I and II (as defined by Fine), hypoxemia and pleural effusion were the significant independent variables observed in the multivariate analysis. In risk classes III, IV and V, further variables were identified. These were diastolic blood pressure, multi-lobe involvement and albumin concentration. When adjusted for these variables, the type-of-hospital variable remained significantly different.

The current investigation was a multi-centre study designed to evaluate the clinical variables, at the first $24 \mathrm{~h}$ of admission to hospital, of a cohort of patients with CAP in four hospitals having different healthcare remits, and to determine the impact on LOS. Although there were no differences with respect to the characteristics of the patients when evaluated on comorbidity, demographical data and class of initial risk (except a higher numbers of smokers in Hospitals C and D) there were significant differences in the duration of hospitalisation. The mortality rate in each hospital evaluated for each initial risk class (as defined by Fine) did not show significant differences.

The authors encountered several factors that correlated (positively or negatively) with the LOS and which corresponded to the initial severity of the illness (PSI, or risk class of Fine), characteristics of the patients, initial antibiotic treatment and the type of hospital. Previous studies [9, 15, 16, 19] had observed (as did the current study) a correlation between greater initial severity of the illness and longer hospitalisation. However, prolonged hospitalisation in low-risk patients was also detected. The Fine scale had been designed and validated, originally, to predict mortality but has since been recommended for decisions regarding hospitalisation. For example, it is proposed that patients in low-risk classes I and II should be treated on an outpatient basis because of the low mortality risk. However, scientific guidelines are not necessarily a substitute for clinical judgement and when this prognostic scale is employed to decide whether to admit a patient, the percentage of admissions is reduced, but not eliminated [20, 21]. This may be due to the limitations associated with specific circumstances of the patients and the underestimation of risk in the younger-aged patients.

Less controversial is the need for admission for the higher risk classes III-V. Therefore, the authors decided to analyse the high-risk sub-group separately and compare the results with the low-risk group, since there is a greater consensus with respect to the decision to admit these high-risk patients.

The advantage of employing multivariate analysis is that it facilitates the selection of independent variables, while discarding those that contain redundant information or confounding variables. The current study also offers the opportunity to assess the influence of hospital type on LOS, since the patient types are similar with respect to clinical circumstances, as well as risk class.

In the mathematical model of the current study the authors encountered five independent variables that best predicted LOS for pneumonia in the overall patient cohort and in the high-risk classes III-V. The independent variables detected were initial hypoxemia, pleural effusion, levels of albumin, number of lobes affected in the initial radiograph and the initial diastolic pressure. The values of $\mathrm{Pa}_{\mathrm{a}} \mathrm{O}_{2}$, albumin and diastolic pressure were inversely related to LOS, while the converse applied to pleural effusion and the number of lobes affected in the initial radiographs. However, in the low-risk classes only two of these clinical factors were detected: hypoxemia and pleural effusion.

Respiratory insufficiency is a morbidity and mortality risk factor that occurs with CAP and is one of the main reasons for hospital admission [22, 23]. HALM et al. [24] observed that the resolution of hypoxemia was the clinical parameter that required more days of hospitalisation to achieve clinical stability. Similarly, the presence of pleural effusion with CAP predisposes a protracted hospitalisation, since this condition requires greater clinical attention, radiological follow-up, thoracocentesis and, eventually, drainage of the thorax. In the current study the authors noted that this was one of the principal motives for hospitalisation and prolonged duration of hospital stay, in risk-class I in Hospital C. Roson et al. [25], in a prospective observational study evaluating hospitalisation on the basis of conventional criteria versus PSI, indicated that $60 \%$ of patients classified as low risk class (I and II of Fine) needed supplemental oxygen or had pleural complications and these were the reasons for which they were hospitalised.

Hypoxemia and pleural effusion are the two clinical factors that can be underestimated in the prognostic scale of Fine, especially in young patients, and account for the admission rates in the risk-classes I and II. In a very recent editorial, HALM and TEIRSTEIN [26] state that all patients with hypoxemia $\left(\mathrm{Pa}, \mathrm{O}_{2}<7.98 \mathrm{kPa}(60 \mathrm{~mm} \cdot \mathrm{Hg})\right.$ while breathing room air) or metastasic disease or empyema should be hospitalised regardless of the score on the Fine scale.

In risk-classes III-V, the effects of hypoxemia and pleural effusion on LOS are associated with three additional clinical variables. Malnutrition is clearly associated with the gravity of the pneumonia and its poorer prognosis [27]. Further, it is more frequent in the elderly and contributes to a slower clinical response to treatment $[27,28]$. Several studies have shown that albumin is a marker of nutritional status and is associated with mortality risk and recovery time of the patient. Multi-lobe involvement and low diastolic blood pressure are risk factors for a complicated clinical course of disease and, as such, it is not surprising that these variables were related to a longer LOS.

The influence of the clinical variables on LOS found in the current study is not unexpected, since they are related to the clinical instability and initial gravity. However, the authors were unable to evaluate the correlations with the time required to reach clinical stability or with the therapeutic response. From this perspective, the association between the initial clinical variables and the resolution of the infection parameters would have provided more insight into clinical and nonclinical factors related to LOS.

With respect to the initial antibiotic regimen employed, univariate analysis indicated that there was a shorter LOS in those patients treated with amoxycillin-clavulanate and macrolides or quinolones. However, this variable was not subsequently selected in the multivariate model. Previous studies that analysed the influence of treatment on the duration of hospitalisation obtained discordant results [29, 30]. Adherence, or not, to the SEPAR guidelines for the 
treatment of CAP did not relate significantly with LOS, as has been reported previously by the authors [31] and others [32].

The type of hospital to which the patient was admitted was a factor predictive of LOS. MCCORMICK et al. [9] had reported that, for a similar risk class, there were differences in hospitals with respect to the duration of stay but without negative effects on several outcomes, such as mortality and re-admission. However, the results reported in the literature are not completely concordant, since some authors highlight possible negative consequences for patients after a shortened stay in hospital [12, 33].

The variability in the duration of hospitalisation with respect to hospital type emphasises the need for objective criteria for the treatment of CAP, so as to reduce the differences in the clinical management of this condition [13, $17,34,35]$. These aspects are beginning to be incorporated in the latest guidelines of the American Thoracic Society [23].

As a limitation of the study, the authors wish to highlight that the mathematical model obtained is not ideal, since the amount of variance explained by the model is not high. However, other variables exist which are not strictly clinical and/or quantifiable and which can influence the LOS. These include hospital inefficiencies, clinical professionals' preferences/idiosyncrasies and difficulties in the management of the patient on an outpatient basis. Another limitation is that the authors did not evaluate the relationships between initial variables and clinical stability and/or clinical response-totherapy separate from the LOS so as to distinguish the impact of other factors, such as social variables, on LOS. Nevertheless, the authors consider that the information gathered is valuable for the clinician and healthcare workers, since it provides a rule of thumb to indicate the probable requirements with respect to length of hospitalisation in CAP.

In summary, in the present study of community-acquired pneumonia the authors observed that in low-risk patients, the length of stay is determined mainly by the level of hypoxemia and pleural effusion, while in the higher risk classes, additional factors, such as multi-lobe involvement, diastolic blood pressure and the albumin concentration, also become significant. The hospital where the patient was admitted exerts an effect on length of stay that is independent of the clinical variables and the severity of the patient's illness on admission. A further area of investigation would be to quantify the influence of variables, such as clinical stability and response to therapy, and length of stay.

Acknowledgement. Editorial assistance was by P.R. Turner of t-SciMed, Reus, Spain.

\section{References}

1. Almirall J, Bolibar I, Vidal J, et al. Epidemiology of community-acquired pneumonia in adults: a populationbased study. Eur Respir J 2000; 15: 757-763.

2. Carretero Gracia JA, Nebreda Mayoral T, Acereda Ridruejo AI, Larumbe Sola Y, Martinez Gutierrez MA, Tierno Sanquirico C. Neumonia adquirida en la comunidad remitida al medio hospitalario. Epidemiología, actitud diagnóstica y terpéutica [Community-acquired pneumonia referred for hospital management. Its epidemiology and the diagnostic and therapeutic approaches]. Arch Bronconeumol 1999; 35: 27-32.

3. Monge V, Gonzalez A. Hospital admissions for pneumonia in Spain. Infection 2001; 29: 3-6.

4. Monge V, San-Martin VM, Gonzalez A. The burden of community-acquired pneumonia in Spain. Eur $J$ Public Health 2001; 11: 362-364.

5. Guest JF, Morris A. Community-acquired pneumonia: the annual cost to the National Health Service in the UK. Eur Respir J 1997; 10: 1530-1534.

6. Fine MJ, Pratt HM, Obrosky DS, et al. Relation between length of hospital stay and costs of care for patients with community-acquired pneumonia. Am J Med 2000; 109: 378385 .

7. Niederman MS, McCombs JS, Unger AN, Kumar A, Popovian R. The cost of treating community-acquired pneumonia. Clin Ther 1998; 20: 820-837.

8. Lave JR, Fine MJ, Sankey SS, Hanusa BM, Weissfeld LA, Kapoor WN. Hospitalized pneumonia. Outcomes, treatment patterns, and costs in urban and rural areas. $J$ Gen Intern Med 1996; 11: 415-421.

9. McCormick D, Fine MJ, Coley CM, et al. Variation in length of hospital stay in patients with community-acquired pneumonia: are shorter stays associated with worse medical outcomes? Am J Med 1999; 107: 5-12.

10. Rosenthal GE, Harper DL, Quinn LM, Cooper GS. Severity-adjusted mortality and length of stay in teaching and nonteaching hospitals. Results of a regional study. JAMA 1997; 278: 485-490.

11. Siegel RE. How long a stay in the hospital is needed for patients with community-acquired pneumonia? Am J Med 2000; 109: 434- 436.

12. Metersky ML, Tate JP, Fine MJ, Petrillo MK, Meehan TP. Temporal trends in outcomes of older patients with pneumonia. Arch Intern Med 2000; 160: 3385-3391.

13. Rhew DC, Tu GS, Ofman J, Henning JM, Richards MS, Weingarten SR. Early switch and early discharge strategies in patients with community-acquired pneumonia: a metaanalysis. Arch Intern Med 2001; 161: 722-727.

14. Weingarten SR, Riedinger MS, Hobson P, et al. Evaluation of a pneumonia practice guideline in an interventional trial. Am J Respir Crit Care Med 1996; 153: 1110-1115.

15. Fine MJ, Auble TE, Yealy DM, et al. A prediction rule to identify low-risk patients with community-acquired pneumonia. N Engl J Med 1997; 336: 243-250.

16. Menendez R, Ferrando D, Valles JM, Martinez E, Perpina M. Initial risk class and length of hospital stay in community-acquired pneumonia. Eur Respir J 2001; 18: 151156.

17. Fine MJ, Medsger AR, Stone RA, et al. The hospital discharge decision for patients with community-acquired pneumonia. Results from the Pneumonia Patient Outcomes Research Team cohort study. Arch Intern Med 1997; 157: 4756.

18. Frias J, Gomis $\mathbf{M}$, Prieto $\mathrm{J}$, et al. Tratamiento antibiótico empírico inicial de la neumonía adquirida en la comunidad [Initial empirical antibiotic treatment of communityacquired pneumonia]. Rev Esp Quimioter 1998; 11: 255261.

19. Masotti L, Ceccarelli E, Cappelli R, Barabesi L, Forconi S. Community-acquired pneumonia in elderly patients and length of hospitalization. Arch Intern Med 2000; 160: 26782679.

20. Atlas SJ, Benzer TI, Borowsky LH, et al. Safely increasing the proportion of patients with community-acquired pneumonia treated as outpatients: an interventional trial. Arch Intern Med 1998; 158: 1350-1356.

21. Marrie TJ, Lau CY, Wheeler SL, Wong CJ, Wandervoot MK, Feagan BG. A controlled trial of a critical pathway for treatment of community-acquired pneumonia. CommunityAcquired Pneumonia Intervention Trial Assessing Levofloxacin (CAPITAL) study investigators. JAMA 2000; 283: 749755.

22. Dorca J, Bello S, Blanquer J, et al. Diagnóstico y tratamiento de la neumonía adquirida en la comunidad. SEPAR. Sociedad Espanola de Neumologia y Cirugia Toracica [The diagnosis and treatment of community-acquired pneumonia]. Arch Bronconeumol 1997; 33: 240-246.

23. Niederman MS, Mandell LA, Anzueto A, et al. Guidelines for the management of adults with community-acquired 
pneumonia. Diagnosis, assessment of severity, antimicrobial therapy, and prevention. Am J Respir Crit Care Med 2001; 163: 1730-1754.

24. Halm EA, Fine MJ, Marrie TJ, et al. Time to clinical stability in patients hospitalized with community-acquired pneumonia: implications for practice guidelines. JAMA 1998; 279: 1452-1457.

25. Roson B, Carratala J, Dorca J, Casanova A, Manresa F, Gudiol F. Etiology, reasons for hospitalization, risk classes, and outcomes of community-acquired pneumonia in patients hospitalized on the basis of conventional admission criteria Clin Infect Dis 2001; 33: 158-165.

26. Halm EA, Teirstein AS. Clinical practice. Management of community-acquired pneumonia. $N$ Engl J Med 2002; 347: 2039-2045.

27. Riquelme $\mathrm{R}$, Torres A, el-Ebiary $\mathrm{M}$, et al. Communityacquired pneumonia in the elderly. Clinical and nutritional aspects. Am J Respir Crit Care Med 1997; 156: 1908-1914.

28. Riquelme R, Torres A, El-Ebiary $\mathrm{M}$, et al. Communityacquired pneumonia in the elderly: A multivariate analysis of risk and prognostic factors. Am J Respir Crit Care Med 1996; 154: 1450-1455.

29. Stahl JE, Barza M, DesJardin J, Martin R, Eckman MH. Effect of macrolides as part of initial empiric therapy on length of stay in patients hospitalized with communityacquired pneumonia. Arch Intern Med 1999; 159: 2576-2580.

30. Dudas V, Hopefl A, Jacobs R, Guglielmo BJ. Antimicrobial selection for hospitalized patients with presumed community-acquired pneumonia: a survey of nonteaching US community hospitals. Ann Pharmacother 2000; 34: 446452.

31. Menendez R, Ferrando D, Valles JM, Vallterra J. Influence of deviation from guidelines on the outcome of communityacquired pneumonia. Chest 2002; 122: 612-617.

32. Marras TK, Chan CK. Use of guidelines in treating community-acquired pneumonia. Chest 1998; 113: 16891694.

33. Rogers WH, Draper D, Kahn KL, et al. Quality of care before and after implementation of the DRG-based prospective payment system. A summary of effects. JAMA 1990 264: 1989-1994.

34. Meehan TP, Weingarten SR, Holmboe ES, et al. A statewide initiative to improve the care of hospitalized pneumonia patients: The Connecticut Pneumonia Pathway Project. Am J Med 2001; 111: 203-210.

35. Rhew DC. Quality indicators for the management of pneumonia in vulnerable elders. Ann Intern Med 2001; 135: 736-743. 